\title{
Porcine adipose-derived stem cells from buccal fat pad and subcutaneous adipose tissue for future preclinical studies in oral surgery
}

\author{
Stefania Niada ${ }^{1,2 \dagger}$, Lorena Maria Ferreira ${ }^{1 \dagger}$, Elena Arrigoni ${ }^{1}$, Alessandro Addis $^{3}$, Marino Campagnol ${ }^{3}$,
} Eugenio Broccaioli ${ }^{1}$ and Anna Teresa Brini ${ }^{1,2^{*}}$

See related commentary by Lopez, http://stemcellres.com/content/5/1/11

\begin{abstract}
Introduction: Adipose-derived stem cells (ASCs) are progenitor cells used in bone tissue engineering and regenerative medicine. Despite subcutaneous adipose tissue being more abundant, the buccal fat pad (BFP) is easily accessible for dentists and maxillofacial surgeons. For this reason, considering the need for preclinical study and the swine as an optimal animal model in tissue engineering applications, we compared the features of porcine ASCs (pASCs) from both tissue-harvesting sites.

Methods: ASCs were isolated from interscapular subcutaneous adipose tissue (Scl) and buccal fat pads of six swine. Cells were characterized for their stemness and multipotent features. Moreover, their osteogenic ability when cultured on titanium disks and silicon carbide-plasma-enhanced chemical vapor-deposition fragments, and their growth in the presence of autologous and heterologous serum were also assessed.

Results: Independent of the harvesting site, no differences in proliferation, viability, and clonogenicity were observed among all the pASC populations. Furthermore, when induced toward osteogenic differentiation, both Scl- and BFP-pASCs showed an increase of collagen and calcified extracellular matrix (ECM) production, alkaline phosphatase activity, and osteonectin expression, indicating their ability to differentiate toward osteoblast-like cells. In addition, they differentiated toward adipocyte-like cells, and chondrogenic induced pASCs were able to increase glycosaminoglycans (GAGs) production over time. When cells were osteoinduced on synthetic biomaterials, they significantly increased the amount of calcified ECM compared with control cells; moreover, titanium showed the osteoinductive effect on pASCs, also without chemical stimuli. Finally, these cells grew nicely in 10\% FBS, and no benefits were produced by substitution with swine serum.
\end{abstract}

Conclusions: Swine buccal fat pad contains progenitor cells with mesenchymal features, and they also osteo-differentiate nicely in association with synthetic supports. We suggest that porcine BFP-ASCs may be applied in preclinical studies of periodontal and bone-defect regeneration.

\section{Introduction}

Dental tissue engineering may now represent an innovative approach to replacing bone and periodontal ligament lost, through the delivery of bioactive molecules and the use of suitable scaffolds and cells. Advanced research in this field leads to rapid progress in tissue repair

\footnotetext{
*Correspondence: anna.brini@unimi.it

${ }^{\dagger}$ Equal contributors

'Dipartimento di Scienze Biomediche, Chirurgiche e Odontoiatriche, Università degli Studi di Milano, Via Vanvitelli, 32, 20129 Milan, Italy ${ }^{2}$ IRCCS Istituto Ortopedico Galeazzi, Milan, Italy

Full list of author information is available at the end of the article
}

and regeneration of oral tissues. Mesenchymal stem cells (MSCs), because of their ability to self-renew, their multidifferentiative potential toward mesodermal cells [1-3], and their plasticity toward cells of ectodermal [4] and endodermal $[5,6]$ origin, are considered proper candidates for these applications. Bone marrow is still the elected source for MSCs [7], although adipose tissue, in the last decade, gained recognition, because adiposederived stem cells (ASCs) can be easily extracted with mild donor-site morbidity or patient discomfort [8]. The first ASCs were isolated from subcutaneous adipose tissue, which is usually discarded after aesthetic surgical

\section{Biomed Central}


procedures. Several studies have also described the presence of ASCs in visceral adipose tissue [9], human orbital fat tissue $[10,11]$, and from special fat pads such as the Hoffa pad [12].

Here, we propose the buccal fat pad (BFP) as a new source for ASCs, which could be of great interest for odontoiatric and maxillofacial surgeons who consider the tissue-engineering approach to be a possible future goal.

The BFP is located between the masseter and buccinators muscles and the ascending mandibular ramus and zygomatic arch [13], and it is easily accessible with a simple surgical procedure under local anesthesia [14]. Since 1977, the BFP has been used in surgery for the treatment of congenital oroantral and/or oronasal diseases [15], congenital cleft palate repair [16], oral submucous fibrosis $[17,18]$, intraoral malignant defects [19], and cheek mucosa defects $[15,20]$. In addition, BFP is a discarded tissue of plastic surgery for cheek reduction. Recent studies showed that human ASCs isolated from the BFP possess all the suitable characteristics for bone tissue engineering, both in vitro [21] and in vivo [22]. Despite the known low immunogenicity of human ASCs, which suggests theoretically their use in preclinical models, we are required to test their safety when implanted in a homologous setting. Considering the potential ability of ASCs in bone regeneration, we have chosen swine as a preclinical model because their bone shares several features with the human bone, such as rate of healing, morphology, anatomy [23], mineral density, and composition [24]. Furthermore, the oral maxillofacial region of these animals is similar in anatomy, development, physiology, pathophysiology, and disease occurrence to the human one [25]. Therefore these animals might be considered appropriate for oral disease models and in orofacial research; they were recently used in preclinical models of dental implants [26-28] and maxillofacial surgery [29-31].

Despite the great variety of supports used in tissue engineering, titanium is widely used in dental surgery, due to its high mechanical and corrosion resistance, as well as its biocompatibility. Interestingly, silicon carbide (SIC), with its hardness and wear-resistance, may be an innovative material suitable to coat metallic implants, giving adequate protection to the material and decreasing the wear rate of the inserted devices. Moreover, SIC obtained by the plasma-enhanced chemical vapor-deposition technique (SIC-PECVD) does not negatively influence any biologic features of human ASCs, in vitro [32], and SIC particles do not give rise to any relevant inflammatory response and do not negatively affect bone growth in vivo [33].

In this study, we described some of the features of ASCs isolated from swine BFP and interscapular subcutaneous adipose tissue from the same animal, and their osteodifferentiation ability in vitro, either in the absence or in the presence of titanium and SIC supports. Finally, pASCs cultured in the presence of autologous and heterologous serum have been also investigated.

\section{Materials and methods}

Isolation of porcine adipose-derived stem cells (pASCs)

Fat tissues and blood samples were collected at the end of preclinical studies approved by the Italian Ministry of Health and were performed at the CRABBC (Biotech Research Centre for Cardiothoracic Applications) (Rivolta d'Adda, CR). All the procedures were carried out in conformity with institutional guidelines in compliance with national (Law 116/92, Authorization n.169/94-A issued December 19, 1994, by the Italian Ministry of Health) and international laws and policies (EEC Council Directive 86/609, OJ L 358. 1, December 12, 1987).

Adipose tissues were collected from subcutaneous interscapular sites (ScIs) and buccal fat pads (BFPs) (Figure 1A, B) from six swine. Porcine adipose-derived stem cells (pASCs) were isolated as previously described. In brief, tissues were enzymatically digested with $0.1 \%$ type I collagenase (225 U/mg; Worthington, Lakewood, NJ, USA) at $37^{\circ} \mathrm{C}$ for 60 minutes. The stromal vascular fraction (SVF) was centrifuged, filtered, and $10^{5}$ cells $/ \mathrm{cm}^{2}$ were plated in DMEM (Sigma-Aldrich, Milan, Italy) supplemented with 10\% FBS, $50 \mathrm{U} / \mathrm{ml}$ penicillin, $50 \mu \mathrm{g} / \mathrm{ml}$ streptomycin, and $2 \mathrm{mM}$ L-glutamine (Sigma-Aldrich) (control medium, CTRL). Cells were maintained at $37^{\circ} \mathrm{C}$ in a humidified atmosphere with $5 \% \mathrm{CO}_{2}$. When cells reached $70 \%$ to $80 \%$ confluence, they were detached with $0.5 \%$ trypsin $/ 0.2 \%$ EDTA (SigmaAldrich) and plated at a density of $5 \times 10^{3}$ cells $/ \mathrm{cm}^{2}$.

\section{Proliferation}

About $5 \times 10^{3}$ cells $/ \mathrm{cm}^{2}$ were maintained in culture for three passages, and regularly detached and counted. Proliferation rate was expressed as doubling time (DT), calculated as follows: $\ln \left(\mathrm{N} / \mathrm{N}_{0}\right) / \mathrm{ln}_{2}$, in which $\mathrm{N}$ represents the number of counted cells, and $\mathrm{N}_{0}$ represents the number of seeded ones.

\section{MTT cell-viability assay}

To test the viability of cells, $1.5 \times 10^{4} \mathrm{pASCs} / \mathrm{cm}^{2}$ were plated in 96-well plates, and monitored at days 1,3 , and 7 . Then $100 \mu \mathrm{l}$ of MTT (3-[4,5 dimethylthiazol-2-yl]-2,5-diphenyltetrazolium bromide; Sigma-Aldrich) (final concentration, $0.5 \mathrm{mg} / \mathrm{ml}$ in DMEM) was added, and cells were maintained for 4 additional hours at $37^{\circ} \mathrm{C}$. Formazan precipitates were solubilized by $100 \%$ DMSO (dimethylsulfoxide; Sigma-Aldrich), and absorbance was read at $570 \mathrm{~nm}$ in a Wallac Victor II plate reader (Perkin Elmer Western Europe, Monza, Italy) [34].

\section{Fibroblast-colony-forming unit assay (CFU-F)}

pASCs were plated in DMEM supplemented with 20\% FBS, $50 \mathrm{U} / \mathrm{ml}$ penicillin, $50 \mu \mathrm{g} / \mathrm{ml}$ streptomycin, and 


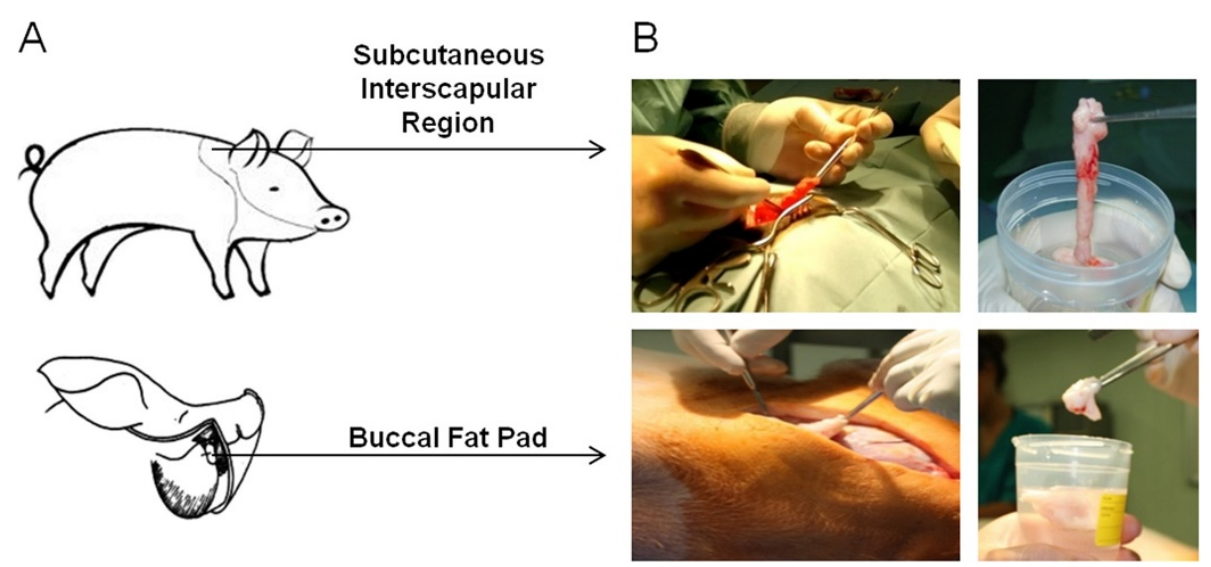

Figure 1 Localization of subcutaneous interscapular and buccal fat pad tissue withdrawal. Anatomic regions of subcutaneous interscapular adipose tissue and buccal fat pad (A). Surgical procedure for tissue collection (B).

$2 \mathrm{~m} M$ L-glutamine, in six-well plates by serial dilution starting from 1,000 cells/well. After 6 days, the medium was replaced, and, at day 10 , cells were washed, fixed in $100 \%$ methanol, and stained with $0.5 \%$ crystal violet (Fluka, Buchs, Switzerland). The frequency of the CFU-F was established by counting individual colonies (of at least 25 cells) compared with the number of seeded cells.

\section{Flow-cytometry analysis}

The pASCs $\left(3 \times 10^{5}\right)$ in $100 \mu \mathrm{l}$ of PBS with $1 \%$ FBS and $0.1 \% \mathrm{NaN}_{3}$ were incubated for 30 minutes on ice with monoclonal antibodies raised against CD14, CD45, CD73, CD90, CD105, and CD271 (Ancell, Bayport, MN, USA). In particular, CD73, CD90, and CD105 were chosen according to the minimal criteria for defining mesenchymal stem cells $[35,36]$. Specific binding was revealed by either streptavidin$\mathrm{PE}$ - or fluorescein isothiocyanate-conjugated sheep antimouse antibody. Samples were acquired by MACSQuant Analyzer (Miltenyi Biotec, Italy), and data were analyzed by using MACSQuantify Software (Miltenyi Biotec).

\section{Osteogenic differentiation}

Cells were maintained in either control or osteogenic medium (OSTEO, DMEM, 10\% FBS, $10 \mathrm{n} M$ dexamethasone, $10 \mathrm{~m} M$ glycerol-2-phosphate, $150 \mu \mathrm{M}$ L-ascorbic acid-2-phosphate, $10 \mathrm{n} M$ cholecalciferol; Sigma-Aldrich) in 24-well plates at the density of $2 \times 10^{4}, 1 \times 10^{4}$, and $5 \times 10^{3} \mathrm{pASCs} /$ well for 7,14 , and 21 days, respectively.

After 7 and 14 days, to determine collagen production, cells were stained with $0.1 \%$ (wt/vol) Sirius Red F3BA in saturated picric acid (Sigma-Aldrich) for 1 hour at room temperature, and then the samples were extracted with $0.1 \mathrm{M} \mathrm{NaOH}$ for 5 minutes [37]. Absorbance was read at $550 \mathrm{~nm}$, as previously. Standard curve of known concentration of calf-skin type I collagen (Sigma-Aldrich) was used to determine the concentration of secreted collagen.
Extracellular matrix (ECM) calcification, at 14 and 21 days, was determined on fixed ASCs stained by $40 \mathrm{~m} M$ Alizarin Red-S (AR-S, pH 4.1; Fluka). Mineral deposition was quantified by incubating the stained sample with $10 \% \mathrm{wt} / \mathrm{vol}$ cetylpyridinium chloride (CPC; SigmaAldrich) in $0.1 \mathrm{M}$ phosphate buffer (pH 7.0) for $15 \mathrm{mi}-$ nutes to extract AR-S. Absorbance was read at $550 \mathrm{~nm}$ with a Wallac Victor II plate reader [38]. To evaluate alkaline phosphatase (ALP) enzymatic activity, both undifferentiated and differentiated ASCs were lysed in $50 \mu \mathrm{l}$ of $0.1 \%$ Triton X-100 and incubated at $37^{\circ} \mathrm{C}$ with $10 \mathrm{~m} M \quad p$-nitrophenylphosphate dissolved in $100 \mathrm{~m} M$ diethanolamine and $0.5 \mathrm{mM} \mathrm{MgCl}$, $\mathrm{pH}$ 10.5. Samples were read at $405 \mathrm{~nm}$, and ALP activity was standardized with respect to the sample protein concentration determined by BCA Protein Assay (Pierce Biotechnology, Rockford, IL, USA).

Osteonectin $(\mathrm{ON})$ expression was also analyzed with Western blot: both undifferentiated and osteo-differentiated cells were lysed in $50 \mathrm{~m} M$ Tris $\mathrm{pH} 8,150 \mathrm{mM} \mathrm{NaCl}$, $1 \%$ Nonidet P40, $0.1 \%$ sodium dodecylsulfate (SDS), supplemented with protease inhibitor cocktail. Then $20 \mu \mathrm{g}$ of protein extracts was resolved by $12.5 \%$ SDSpolyacrylamide gel (Bio-Rad Laboratories), electrotransferred onto HybondTM-ECLTM extra nitrocellulose membrane (GE Healthcare), and probed with either mouse anti-ON (1:100 dilution; Santa Cruz Biotechnology), and mouse anti- $\beta$-actin (1:5,000 dilution, Sigma-Aldrich). Specific proteins were revealed by horseradish peroxidase (HRP)-conjugated secondary antibodies (GE Healthcare) and the ECL Western Blotting Analysis System Kit (GE Healthcare), according to the manufacturer's protocol.

\section{Adipogenic differentiation}

Porcine ASCs were induced to differentiate toward the adipogenic lineage, as previously described [21]. In brief, 
$1.5 \times 10^{4} \mathrm{pASCs} / \mathrm{cm}^{2}$ were plated and cultured in control medium supplemented with $1 \mu M$ dexamethasone, $10 \mu \mathrm{g} / \mathrm{ml}$ insulin, $500 \mu M$ 3-isobutyl-1-methyl-xanthine, and $200 \mu M$ indomethacin (Sigma-Aldrich). At 14 days later, cells were fixed in $10 \%$ neutral buffered formalin for 1 hour and stained with fresh Oil Red $O$ solution (20 $\mathrm{mg} / \mathrm{ml}$ (wt/vol) Oil Red $\mathrm{O}$ in 60\% isopropanol) for 15 minutes. Lipid vacuoles were quantified by extraction with $200 \mu \mathrm{l}$ of $100 \%$ isopropanol for 10 minutes and reading the absorbance of $50 \mu \mathrm{l}$ at $490 \mathrm{~nm}$ with the Wallac Victor II plate reader.

\section{Chondrogenic differentiation}

Then $5 \times 10^{5}$ pASCs were cultured in micromasses in chondrogenic medium (DMEM supplemented with $1 \%$ FBS, $100 \mathrm{n} M$ dexamethasone, $110 \mathrm{mg} / \mathrm{L}$ sodium pyruvate, $150 \mu M$ L-ascorbic acid-2-phosphate, $1 \times$ insulintransferrin selenium (ITS) and $10 \mathrm{ng} / \mathrm{ml}$ TGF- $\beta 1$ ) for 21 days. Glycosaminoglycans (GAGs) production was assessed with dimethylmethylene blue (DMMB) assay, as previously described $[39,40]$. In brief, micromasses were digested at $56^{\circ} \mathrm{C}$ overnight by $100 \mu \mathrm{l}$ of $50 \mu \mathrm{g} / \mathrm{ml}$ proteinase $\mathrm{K}$ in $100 \mathrm{mM} \mathrm{K} \mathrm{HPO}_{4}(\mathrm{pH} \mathrm{8.0)}$. After 10 minutes at $90^{\circ} \mathrm{C}$ to inactivate the enzyme, the samples were spun at $14,000 \mathrm{~g}$ for 10 minutes, and each supernatant was collected for GAGs and DNA quantification. The samples were then incubated at room temperature in $40 \mathrm{mM}$ glycine/ $\mathrm{NaCl}(\mathrm{pH} \mathrm{3})$ with $16 \mathrm{mg} / \mathrm{ml} \mathrm{DMMB}$, and the absorbance was read at $500 \mathrm{~nm}$ with the Wallac Victor II plate reader. The amount of GAGs was determined with respect to known concentrations of chondroitin sulfate (Sigma-Aldrich) and normalized on total DNA content determined as described later. Then $0.2 \mu \mathrm{g} / \mathrm{ml}$ Hoechst 33258 was added to the samples for 1 minute at room temperature, fluorescence was measured (excitation at 340 to $370 \mathrm{~nm}$; emission, 440 to $460 \mathrm{~nm}$ ), and DNA concentration for each sample determined with respect to the standard curve of salmon sperm DNA.

\section{ASC culture and osteogenic differentiation on biomaterials}

Both ScI- and BFP-pASCs were seeded at $5 \times 10^{3} / \mathrm{cm}^{2}$ on titanium disk (kindly provided by Permedica S.p.A., Merate, Italy) and silicon carbide-plasma-enhanced chemical-vapor deposition (SIC) fragments (kindly provided by CETEV, Centro Tecnologico del Vuoto, Carsoli, AQ, Italy) either in CTRL or OSTEO medium. To determine cells adhering to the biomaterials, both undifferentiated and differentiated pASCs for 21 days, were lysed in $0.1 \%$ Triton X-100, and protein concentration was determined by BCA Protein Assay, as described earlier. Meanwhile, in adjacent wells, calcified ECM deposition was determined, and compared with the one produced by plastic-adherent (PA) cells.

\section{Porcine serum collection}

Then $10 \mathrm{ml}$ of blood from each animal was allowed to clot for 30 to 45 minutes at $37^{\circ} \mathrm{C}$ and then transferred at $4^{\circ} \mathrm{C}$ for 30 minutes. After centrifugation $(1,000 \mathrm{~g}$ for 10 minutes), sera were collected under sterile conditions [41] and maintained at $-20^{\circ} \mathrm{C}$ until their use.

\section{Statistical analysis}

Data are expressed as mean \pm SEM. Statistical analyses were performed by using Student $t$ test. Differences were considered significant at $P<0.05$.

\section{Results}

\section{Comparison between porcine ASCs isolated from two different body sites}

We collected different amounts of subcutaneous interscapular adipose tissue (ScI-pASCs) and buccal fat pad (BFP-pASCs) from six swine, as indicated in Table 1. We isolated $5.5 \times 10^{4} \pm 3.3 \times 10^{4} \mathrm{ScI}-\mathrm{pASCs} / \mathrm{ml}$ and $3.0 \times 10^{4} \pm 9.3 \times 10^{3}$ BFP-pASCs $/ \mathrm{ml}$ of raw tissue. $\mathrm{pASCs}$ adhered nicely to tissue-culture plates, and in a week, they began to proliferate, showing an MSC-typical fibroblast-like morphology (Figure 2C). In details, the doubling times (DT) of the two cell populations were constant, and no significant differences were observed between ScI-pASCs and BFP-pASCs. Indeed, the mean DT was of about $82.9 \pm 11.5$ hours for ScI-pASCs and $72.5 \pm 8.2$ hours for BFP-pASCs (Figure 2A). Furthermore, cell viability was maintained for all the pASC populations analyzed (Figure $2 \mathrm{~B}$ ), and their proliferation trend was quite stable.

Porcine ASCs held a strong clonogenic ability that was maintained along passage I to IV (Figure 2D): about $10.1 \% \pm 1.4 \%$ of ScI-pASCs and $8.9 \% \pm 1.5 \%$ of BFPpASCs produced CFU-F. Moreover, both pASC populations were immunophenotyped, and a FACS analysis of both cells derived from two animals is shown in Figure 3. Both ScI- and BFP-pASCs appeared similar in size and granularity (upper panels), and both cell types expressed CD90 (middle panels), whereas the CD271 was not detectable (lower panels), as CD14 and CD45 (data not shown). Unfortunately, no cross-reactivity was found on both ScI- and BFP-pASCs for CD73 and CD105 (data not shown).

Table 1 pASC source

\begin{tabular}{cccccc}
\hline & & Gender & Age & $\boldsymbol{n}$ & Raw adipose tissue $(\mathbf{m l})$ \\
\hline pASCs & Scl & $3 \hat{\sigma}, 3 q$ & $\geq 4$ months & 6 & $12.3 \pm 3.6$ \\
& BFP & $3 \hat{\delta}, 3 q$ & $\geq 5$ months & 6 & $5.7 \pm 1.5$ \\
\hline
\end{tabular}

Gender, age of the animals, amount of harvested fat from subcutaneous interscapular adipose tissue ( $\mathrm{Scl}$ ), and buccal fat pad (BFP). Data are expressed as mean \pm SEM. 

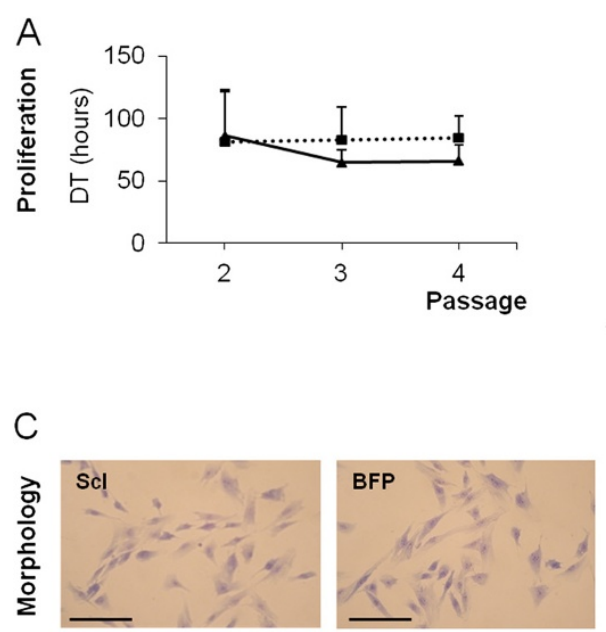
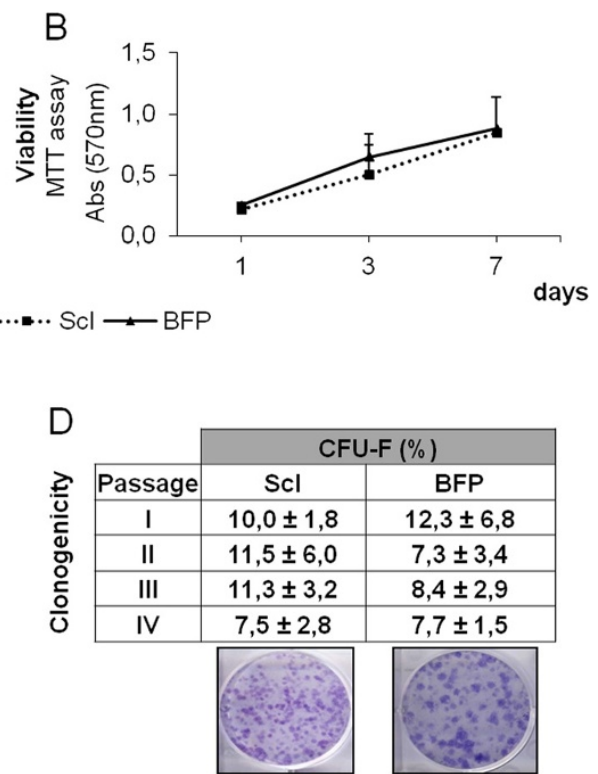

Figure 2 Stemness features of Scl- and BFP-pASCs. Cell proliferation expressed as doubling time (DT, hours) of ASCs from II to IV passage (A). Viability assessed by MTT assay at days 1, 3, and 7 (B). Morphology of Scl-pASCs and BFP-pASCs (optical microscopy, 200x magnification; scale bar, $100 \mu \mathrm{m})$ (C). Clonogenicity from passage I to IV expressed as colony-forming units (CFU-F) percentage (ratio of number of colonies/number of plated cells $\times 100)(\mathbf{D}$, upper panel). Data are expressed as mean \pm SEM $(n=6)$. Representative Scl-pASCs and BFP-pASCs plates stained with crystal violet (D, lower panel).

\section{Osteogenic, adipogenic, and chondrogenic differentiation of Scl-pASCs and BFP-pASCs}

Osteogenic differentiated ScI- and BFP-pASCs significantly increased the production of bone-specific markers, such as collagen (Figure 4A,B), calcified ECM (Figure 4C,D), alkaline phosphatase (ALP) activity (Figure 4E), and osteonectin (ON, Figure 4F), compared with undifferentiated cells.

Although BFP-pASCs showed a slightly lower basal level of collagen deposition with respect to ScI-pASCs, these differences at 7 and 14 days were not significant. Collagen level significantly increased by about $42 \%$ and $310 \%$ in 7 days osteoinduced ScI- and BFP-pASCs, respectively, compared with undifferentiated cells (CTRL); this upregulation further increased for ScI-pASCs (+87\%) and was maintained $(+254 \%)$ for BFP-pASCs (Figure 4A,B). In addition, osteodifferentiated pASCs produced abundant amounts of calcified ECM, and in 2 weeks, ECM calcification increased by about $118 \%$ and $116 \%$ for ScI- and for BFP-pASCs, respectively (Figure 4C,D). Meanwhile, ALP activity was also determined: after 1 week of culture, we observed an upregulated ALP activity in both osteodifferentiated ScI and BFP cells, compared with undifferentiated ones, with increases of $126 \%$ and $201 \%$, respectively (Figure 4E). This trend was also maintained after 14 days (Figure 4E). In Figure 4F, we show that osteonectin $(\mathrm{ON})$ expression is induced of about 336\% and 306\% in osteodifferentiated ScI- and BFP-pASCs, respectively.
The multidifferentiative ability of BFP-pASCs was further tested and compared with ScI-pASCs. At first, as shown in Figure 5A, adipogenic differentiation is observed after 14 days: the morphology of both cell types is remarkably modified, from the usual fibroblast-like shape to a round one, with a cytoplasmic accumulation of lipid vacuoles (upper panel). Oil Red O staining (Figure 5A, middle panel), and its quantification proved that both cell types similarly differentiate $(+121 \%$ for ScI-pASCs and $+130 \%$ for BFP-pASCs, with respect to control cells) (Figure 5A, lower panel).

Then we also determined GAGs content in both chondrogenic differentiated pASCs for 1, 2, and 3 weeks. We observed an increase of GAGs deposition during that time. Indeed, after 14 days, the GAG content, with respect to 7 days, was more abundant at $56 \%$ and $45 \%$ in ScI- and BFP-pASCs, respectively, and it was further upregulated after 21 days $(+184 \%$ and $+149 \%$ for ScI- and BFP-pASCs, respectively) (Figure 5B). We conclude that both pASCs display in vitro the multipotent feature of mesenchymal stem cells.

\section{pASCs on biomaterials}

pASCs cultured for 21 days on biomaterials, both in the presence and in the absence of osteogenic stimuli, efficiently adhered to them; indeed, no significant differences were observed between the protein concentrations 


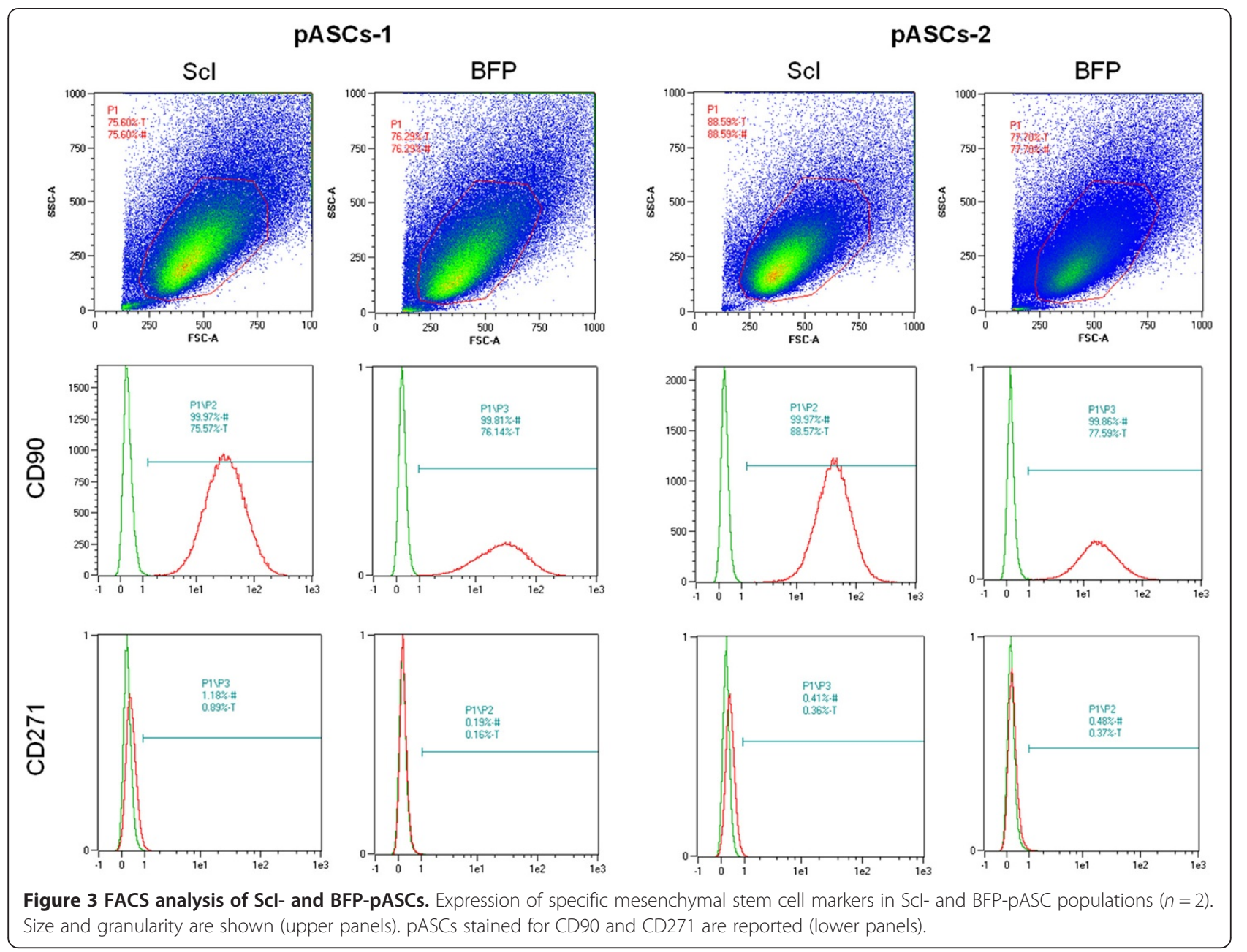

of either plastic adherent cells or scaffold-associated ones. This indirect evidence is shown in Figure 6B. ScIand BFP-pASCs cultured for 7 days on the supports are alive and tightly laid on them when observed by confocal microscopy (data not shown).

Both pASCs, cultured on biomaterials, differentiated toward cells of the osteogenic lineage. Indeed, pASCs seeded on TIT, and osteodifferentiated, deposited an increased amount of calcified ECM of about $46 \%$ and $37 \%$ for ScI- and BFP-pASCs, respectively, compared with CTRL cells; similarly, ScI- and BFP-pASCs on SIC, increased ECM deposition of $90 \%$ and $200 \%$, respectively, compared with CTRL cells.

Moreover, TIT is osteoinductive for pASCs; we quantified an increase of calcified ECM of about $91 \%$ in CTRL ScI-pASCs, and of about 234\% in CTRL BFP-pASCs, compared with plastic-adherent cells (Figure 6A).

\section{Culture of pASCs in porcine serum}

Considering porcine ASCs useful in preclinical models, we compared their behavior when they were cultured in medium supplemented with porcine serum, 5\% autologous (AS), and 5\% heterologous sera (HS), and with $10 \%$ FBS (standard condition). Cells did not proliferate as fast as when maintained in standard condition. In more detail, the number of pASCs collected after 3 weeks was about $4.4 \% \pm 2.4 \%$ compared with cells grown in standard condition and set as $100 \%$ (data not shown). After 7 days, the presence of either autologous or heterologous sera did not allow a rapid cell growth. However, after 21 days, pASCs grown in the presence of HS increased their number with respect to pASCs cultured in AS (Figure 7). As depicted also in Figure 7, all ASCs, grown in the presence of autologous or heterologous serum, aggregated in small clusters and changed morphology, becoming smaller and rounder compared with cells cultured in $10 \%$ FBS.

\section{Discussion}

We investigated the possibility of isolating porcine ASCs from Buccal Fat Pads (BFP-pASCs), which have similar stemness features to the ones isolated from 
A

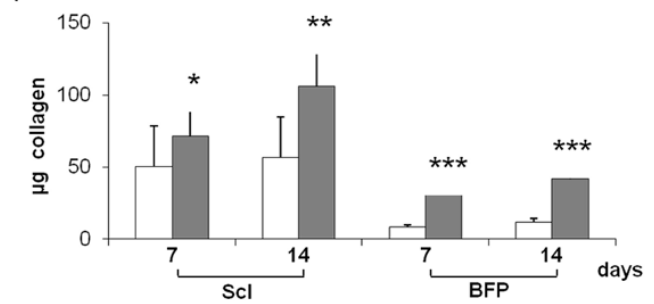

C

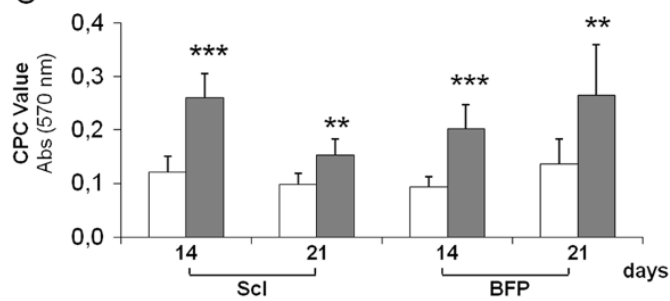

E

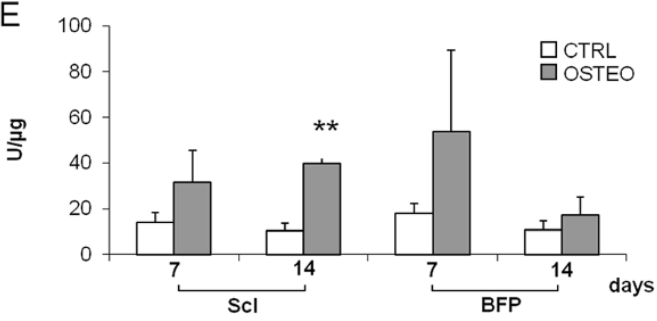

$\mathrm{B}$

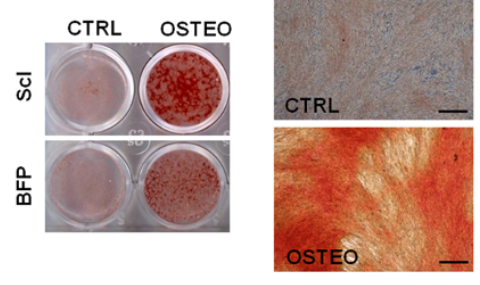

D
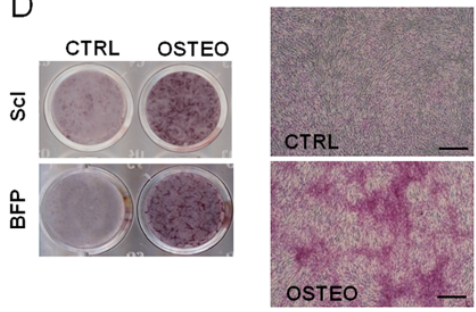

$\mathrm{F}$
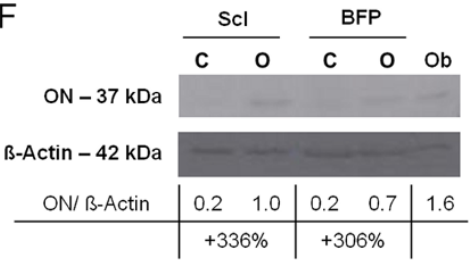

Figure 4 Osteogenic potential of pASCs. Quantification of collagen (A), calcified extracellular matrix (ECM) deposition (C), alkaline phosphatase (ALP) activity (E) in undifferentiated (CTRL, white bars), and osteo-differentiated (OSTEO, dark bars) SCl- and BFP-pASCs; data are expressed as mean \pm SEM $(n=12)$. OSTEO versus CTRL ${ }^{*} P<0.05 ;{ }^{*} P<0.01 ;{ }^{* *} P<0.001$. Images of Scl- and BFP-pASC wells stained with Sirius Red (B, left panel) and Alizarin Red-S (D, left panel) and representative microphotographs of Scl-pASCS (B, D, right panel, optical microscopy, 40x magnification; scale bar, $200 \mu \mathrm{m})$. Osteonectin expression of Scl-pASCs and BFP-pASCs analyzed with Western blot; its quantification, normalized to $\beta$-actin, is also indicated (F).

subcutaneous tissue (ScI-pASCs), previously characterized [34]. Human BFP-ASCs might be quite easily applied in oral tissue engineering, because this tissue is rapidly accessible by dentists and maxillofacial surgeons [14]. However, before moving to the clinic, it is mandatory to perform approved preclinical studies to validate the safety and efficacy of cellular therapies. The most used largeanimal model of human oral bone defects is swine [31,42], because these animals present a healing potential comparable to that of the human. Several studies have been conducted by using stem cells in oral diseases and orofacial research: Wilson et al. [31] investigated bone regeneration in the pig mandible ramus by either local or systemic ASCs injection, concluding that both treatments accelerate the healing process, without any significant difference between the two routes of administration. In another study, similar results were obtained combining decidua stem cells with a $\beta$-TCP scaffold in a minipig model [43].

Here we compared pASCs derived from two different body areas and evaluated their behavior in vitro to identify a convenient source for future preclinical studies.
BFP-pASCs were very similar to ScI-pASCs. Although the cellular yield of the porcine ASCs was lower than the human one [44], after 30 days in culture, we could have been able to obtain a homogeneous populations of about $10^{8}$ to $10^{9}$ cells, with still a pronounced clonogenic ability.

Both cell populations, analyzed at passage 4 , were $\mathrm{CD}^{+}, \mathrm{CD} 271^{-}, \mathrm{CD} 45^{-}$, and $\mathrm{CD} 14^{-}$. These results are similar to the ones on porcine MSCs from different tissues [45], and to our results on human mesenchymal stem cells from the Bichat fat pad that express CD90, CD73, and CD105 [21], as defined for human mesenchymal stromal cells $[35,36]$.

In conclusion, both cell populations were highly positive for CD90, one of the main MSC surface antigens, whereas no cross-reactivity has been observed for CD73 and CD105. Although limited, these results are consistent with the ones obtained with porcine MSCs from bone marrow [46].

Furthermore, by a molecular approach of RT-PCR, we have preliminary data on the expression of Kruppel-like 


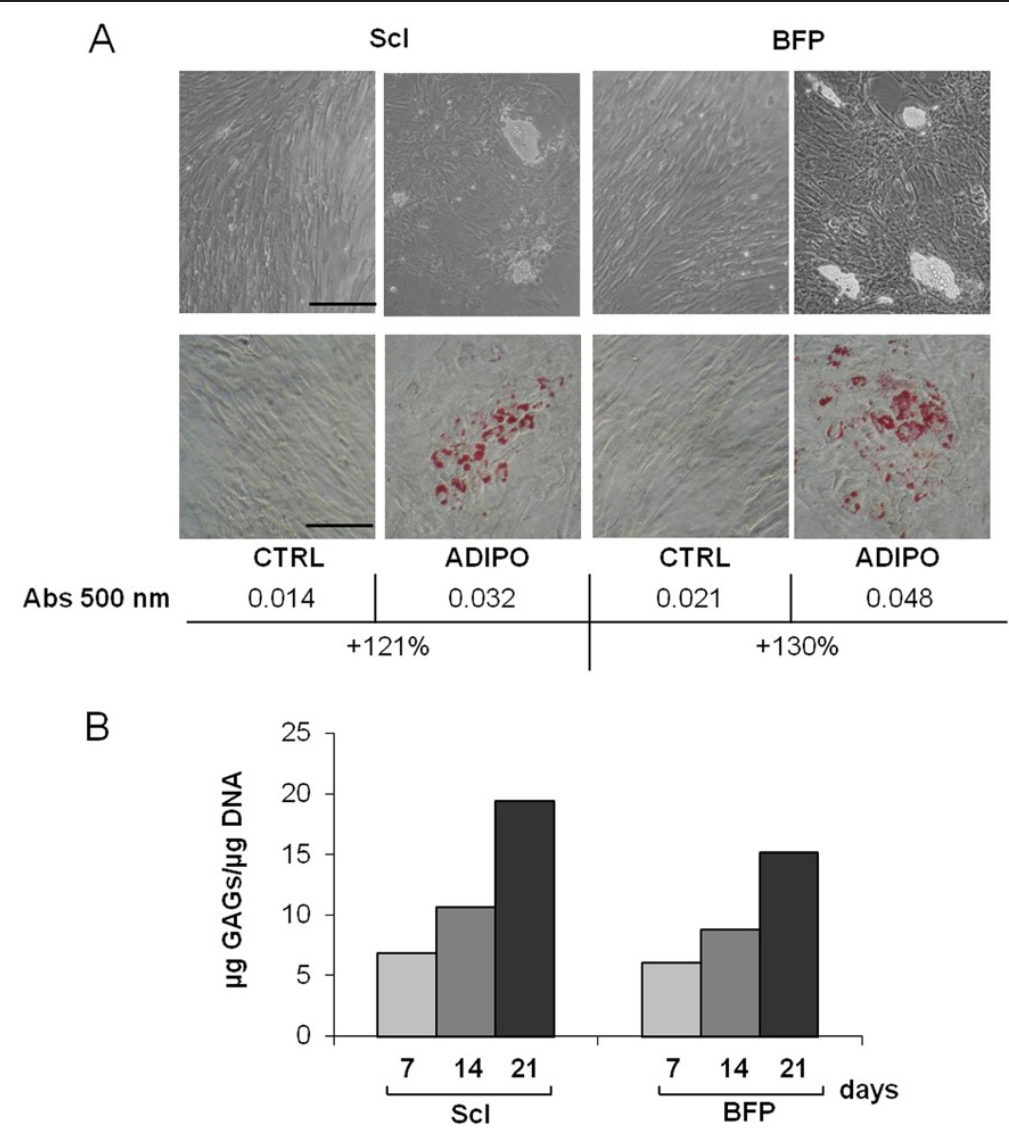

Figure 5 Adipogenic and chondrogenic potential of pASCs. Microphotographs of BFP-pASCs and Scl-pASCs maintained for 14 days in control (CTRL) and adipogenic medium (ADIPO; 200× magnification; scale bar, $50 \mu \mathrm{m}$ ), both during culture (A, upper panels) and after lipid vacuoles staining by Oil Red O (B, middle panels). Quantification of lipid vacuoles formation by Oil Red O extraction is shown in $\mathbf{A}$, lower panel. Quantification of glycosaminoglycans (GAGs) production normalized on DNA content in CHONDRO-pASCs after 7, 14, and 21 days of differentiation in pellet culture (B)

factor 4 (Klf-4), a marker of immature stem cells involved in the control of cell multipotency in many development-related processes and in the maintenance of stem cell-associated properties [47]. The mRNA expression levels in BFP-pASCs are comparable to the ones in human-ASCs. We consider this result interesting, because we recently showed that Klf- 4 expression in hASCs seems to be related to the cell proliferation, clonogenic ability, and differentiative potential, and to be downregulated by the pathologic condition (obesity) of patients from which cells were isolated [48].

Besides, all the porcine BFP-ASCs, grown in the presence of inductive stimuli, nicely increased both osteogenic and adipogenic features, as already described for subcutaneous porcine ASCs [34,49,50]. At last, both populations are able to progressively depose GAGs during 3D culture when induced to chondro-differentiate. Altogether, these results suggest our claim that swine buccal fat pad contains progenitor cells of the mesenchymal stromal cell family, similar to the human ones.
Because these cells could be used in preclinical studies of tissue engineering, and their interaction with appropriate supports is essential, we evaluated the ability of both pASCs to grow and differentiate onto two synthetic scaffolds: the former, a widely used biomaterial in dental surgeries (titanium), and the latter, a promising candidate for the coating of some portions of implant (SiC-PECVD). Like human ASCs [32], pASCs adhere and differentiate on both scaffolds. Moreover, the osteoinductive properties of titanium on hASCs [32], were also observed on both porcine progenitor cells, whereas SIC-PECVD did not modulate their osteogenic differentiation.

Next, testing porcine autologous or heterologous sera, we detected that pASCs proliferated slower than cells cultured in the presence of FBS, and they dramatically stopped growing, changed morphology, and aggregated in clusters. These data are consistent with previous data by Schwarz et al. [41], in which equine ASCs cultured with autologous serum proliferate less than with FBS. Differently, our results are in contrast with data obtained 


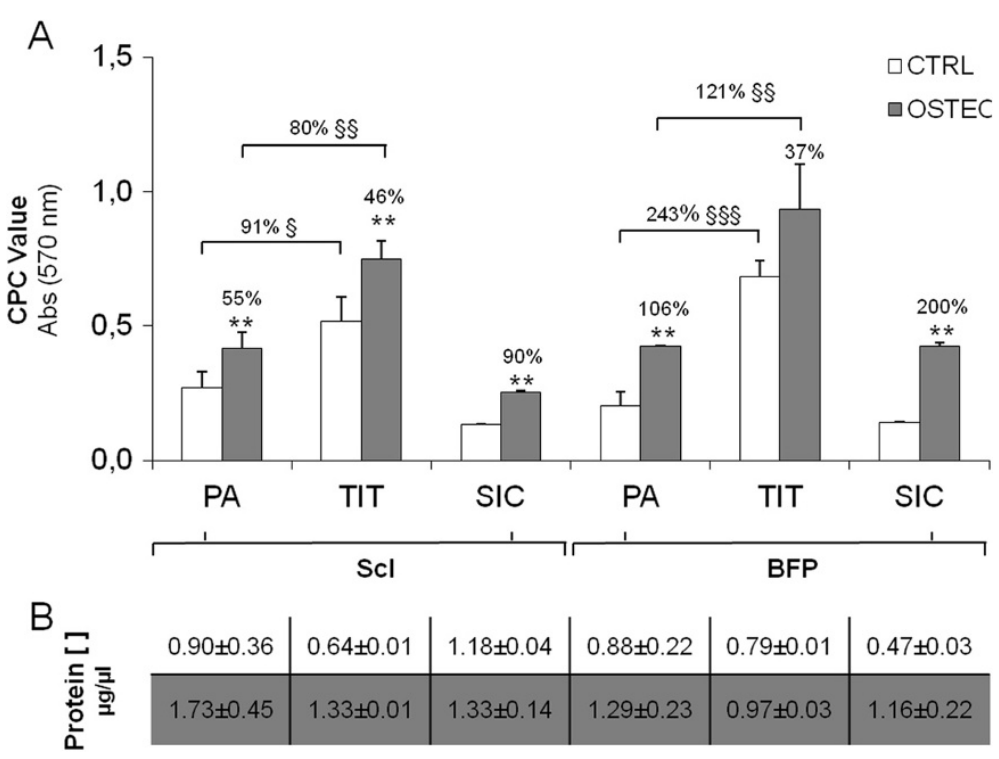

Figure 6 Influence of biomaterials on pASC osteogenic differentiation. Calcified ECM deposition in undifferentiated (CTRL, white bars) and osteogenic-differentiated (OSTEO, dark bars) Scl- and BFP-pASCs, cultured for 21 days on monolayer (plastic adherent, PA), or seeded on titanium disks (TIT) or on silicon carbide-plasma-enhanced chemical vapor deposition (SIC) fragments (A). Protein concentration of pASCS cultured either in monolayer or adhering to biomaterial both in CTRL (white row) and OSTEO (dark row) is reported in panel (B). Data are expressed as mean \pm SEM $(n=3)$. OSTEO versus CTRL: ${ }^{* *} P<0.01$; TIT versus PA; ${ }^{\S} P<0.05 ;{ }^{\S \S} P<0.01 ;{ }^{\S \S \S} P<0.001$.

with human ASCs, in which it has been shown that the use of autologous serum favors or does not influence ASCs proliferation $[21,51,52]$. Nevertheless, Kurita et al. [53] showed that among four human ASC populations, only one proliferates faster when cultured with autologous serum. This discrepancy has also been observed for

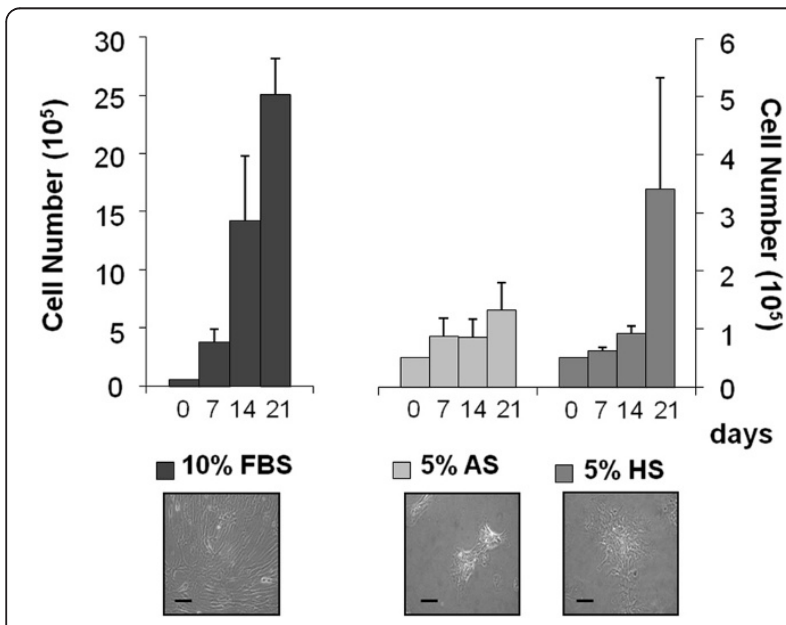

Figure $7 \mathrm{pASCs}$ cultured in media supplemented with porcine sera. PASCs were grown for 7, 14, and 21 days, in DMEM supplemented with $10 \%$ FBS or $5 \%$ autologous or heterologous serum. Data are expressed as mean \pm SEM $(n=4)$. Microphotographs of Scl-pASCs in culture for 21 days (lower panel, optical microscopy, 100x magnification, scale bar $50 \mu \mathrm{m})$. AS, autologous serum; FBS, fetal bovine serum; HS, heterologous serum. human bone marrow stem cells [54-57], suggesting that other factors may influence cell growth. This issue requires further investigation to be clarified, although we have shown that both pASCs behaved similarly.

\section{Conclusions}

Our data suggest that the buccal fat pad might be a novel source of MSCs. This region contains a population of progenitor cells with stemness features that are able to differentiate in vitro and also are associated with synthetic supports. This is quite relevant for maxillofacial and dental surgeons, because for them, human BFP is an easily reachable and convenient area. Human ASCs have been isolated from small specimens of BFP ( $1 \mathrm{ml}$ of tissue), and they are similar to the most known ScI-ASCs [21,58]. Data about human cells and previous data on pASCs $[2,8,34]$ are consistent with our work, indicating that BFP-ASCs are comparable with ASCs isolated from human and porcine subcutaneous tissue. Although it could be debatable to isolate cells from a very small fat pad, we think that progenitor cells derived from a nearby area of the defect could push toward a proper use of BFP-ASCs in oral clinical studies. The natural localization of BFP-ASCs could make them more prone to respond to stimuli naturally secreted in the mouth, as we previously observed regarding the osteoinductive properties on human BFP-ASCs of amelogenin, the most abundant enamel matrix protein [21]. For preclinical test, the low immunogenicity of mesenchymal stem cells might be exploited by using heterologous 
porcine BFP-ASCs, because in swine, the buccal fat pad is not so easily accessible as in the humans.

Our data support future clinical applications of human BFP-ASCs in a tissue-engineering approach for oral and maxillofacial diseases, and we suggest swine as a convenient preclinical model to test new bioconstructs.

\section{Abbreviations}

AR-S: Alizarin red-s; AS: Autologous serum; ASCs: Adipose-derived stem cells; BFP: Buccal fat pad; CFU-F: Fibroblast-colony-forming unit;

CPC: Cetylpyridinium chloride; CTRL: Control medium; DMEM: Dulbecco modified Eagle medium; DMSO: Dimethyl sulfoxide; DT: Doubling time; ECM: Extracellular matrix; EDTA: Ethylenediaminetetraacetic acid; FBS: Fetal bovine serum; HS: Heterologous serum; MSCs: Mesenchymal stem cells; MTT: 3-[4,5 dimethylthiazol-2-yl]-2,5-diphenyltetrazolium bromide; OSTEO: Osteogenic medium; Scl: Subcutaneous interscapular; SEM: Standard error of the mean; SIC: Silicon carbide; SIC-PECVD: Silicon carbide-plasmaenhanced chemical vapor deposition; SVF: Stromal vascular fraction; TIT: Titanium.

\section{Competing interests}

The authors declare that they have no competing interests.

\section{Authors' contributions}

SN and LMF participated in the conception and design of the study, collecting samples, and their analysis and interpretation. They carried out all the cell isolation, expansion, and characterization. They carried out FACS and biochemical analyses, and gene and protein expression tests. Both authors have been involved in the writing process, and their critical intellectual role has been determinant. EA made substantial contributions to acquisition, analysis, and interpretation of data, and she carried out the statistical analysis. She has also been involved in manuscript writing. AA made substantial contributions to design, analysis, and interpretation of data, and she was responsible of animal handling and surgery. MC has made substantial contributions to the in vivo part of the study and in the in vivo study design, collecting biologic specimens, acquiring data, and their interpretation. EB contributed to the conception and design of the study, and interpretation of the data and he was involved in drafting the manuscript and revising it critically for important intellectual content. ATB contributed to conception and design of the study, analysis and interpretation of the data, and she was involved in drafting the manuscript and in the study's funding. She also gave final approval of the version to be published, and she agreed to be accountable for all aspects of the work in ensuring that questions related to the accuracy or integrity of any part of the work are appropriately investigated and resolved. All authors read and approved the final manuscript.

\section{Acknowledgements}

This study was partially supported by the Italian Ministry of Health (2007-656853), University grant FIRST-2008 and RC-2044 IRCCS Istituto Ortopedico Galeazzi (Milano). The authors thank Dr. S. Fenu (Fondazione INGM, Istituto Nazionale di Genetica Molecolare, Milano) for his valuable help with confocal microscopy.

\section{Author details}

${ }^{1}$ Dipartimento di Scienze Biomediche, Chirurgiche e Odontoiatriche, Università degli Studi di Milano, Via Vanvitelli, 32, 20129 Milan, Italy. ${ }^{2}$ IRCCS Istituto Ortopedico Galeazzi, Milan, Italy. ${ }^{3} \mathrm{CRABCC}$ srl, Rivolta d'Adda, Cremona, Italy.

Received: 9 May 2013 Revised: 7 October 2013

Accepted: 26 November 2013 Published: 11 December 2013

\section{References}

1. Lee JH, Kemp DM: Human adipose-derived stem cells display myogenic potential and perturbed function in hypoxic conditions. Biochem Biophys Res Commun 2006, 341:882-888.

2. De Girolamo L, Sartori MF, Arrigoni E, Rimondini L, Albisetti W, Weinstein RL, Brini AT: Human adipose-derived stem cells as future tools in tissue regeneration: osteogenic differentiation and cell-scaffold interaction. Int J Artif Organs 2008, 31:467-479.
3. Yu G, Floyd ZE, Wu X, Halvorsen YD, Gimble JM: Isolation of human adipose-derived stem cells from lipoaspirates. Methods Mol Biol 2011, 702:17-27.

4. Fujimura J, Ogawa R, Mizuno H, Fukunaga Y, Suzuki H: Neural differentiation of adipose-derived stem cells isolated from gfp transgenic mice. Biochem Biophys Res Commun 2005, 333:116-121.

5. Banas A, Teratani T, Yamamoto Y, Tokuhara M, Takeshita F, Quinn G, Okochi $\mathrm{H}$, Ochiya T: Adipose tissue-derived mesenchymal stem cells as a source of human hepatocytes. Hepatology 2007, 46:219-228.

6. Timper K, Seboek D, Eberhardt M, Linscheid P, Christ-Crain M, Keller U, Muller B, Zulewski H: Human adipose tissue-derived mesenchymal stem cells differentiate into insulin, somatostatin, and glucagon expressing cells. Biochem Biophys Res Commun 2006, 341:1135-1140.

7. Caplan Al: Mesenchymal stem cells. J Orthop Res 1991, 9:641-650.

8. Zhu Y, Liu T, Song K, Fan X, Ma X, Cui Z: Adipose-derived stem cell: a better stem cell than BMSC. Cell Biochem Funct 2008, 26:664-675.

9. Baglioni S, Cantini G, Poli G, Francalanci M, Squecco R, Di Franco A, Borgogni E, Frontera S, Nesi G, Liotta F, Lucchese M, Perigli G, Francini F, Forti G, Serio M, Luconi M: Functional differences in visceral and subcutaneous fat pads originate from differences in the adipose stem cell. Plos One 2012, 7:e36569.

10. Korn BS, Kikkawa DO, Hicok KC: Identification and characterization of adult stem cells from human orbital adipose tissue. Ophthal Plast Reconstr Surg 2009, 25:27-32.

11. Ho JH, Ma WH, Tseng TC, Chen YF, Chen MH, Lee OK: Isolation and characterization of multi-potent stem cells from human orbital fat tissues. Tissue Eng Part A 2011, 17:255-266.

12. Jurgens WJ, van Dijk A, Doulabi BZ, Niessen FB, Ritt MJ, van Milligen FJ, Helder MN: Freshly isolated stromal cells from the infrapatellar fat pad are suitable for a one-step surgical procedure to regenerate cartilage tissue. Cytotherapy 2009, 11:1052-1064.

13. Abad-Gallegos M, Figueiredo R, Rodriguez-Baeza A, Gay-Escoda C: Use of Bichat's buccal fat pad for the sealing of orosinusal communications: a presentation of 8 cases. Med Oral Patol Oral Cir Bucal 2011, 16:e215-e219.

14. Meyer E, Liebenberg SJ, Fagan JJ: Buccal fat pad: a simple, underutilised flap. S Afr J Surg 2012, 50:47-49.

15. Egyedi P: Utilization of the buccal fat pad for closure of oro-antral and/or oro-nasal communications. J Maxillofac Surg 1977, 5:241-244.

16. Levi B, Kasten SJ, Buchman SR: Utilization of the buccal fat pad flap for congenital cleft palate repair. Plast Reconstr Surg 2009, 123:1018-1021.

17. Saravanan K, Narayanan V: The use of buccal fat pad in the treatment of oral submucous fibrosis: a newer method. Int J Dent 2012, 2012:935135.

18. Surej KL, Kurien NM, Sakkir N: Buccal fat pad reconstruction for oral submucous fibrosis. Natl J Maxillofac Surg 2010, 1:164-167.

19. Chakrabarti J, Tekriwal R, Ganguli A, Ghosh S, Mishra PK: Pedicled buccal fat pad flap for intraoral malignant defects: a series of 29 cases. Indian J Plast Surg 2009, 42:36-42.

20. Ferrari S, Ferri A, Bianchi B, Copelli C, Magri AS, Sesenna E: A novel technique for cheek mucosa defect reconstruction using a pedicled buccal fat pad and buccinator myomucosal island flap. Oral Oncol 2009, 45:59-62.

21. Broccaioli E, Niada S, Rasperini G, Ferreira LM, Arrigoni E, Yenagi V, Brini AT: Mesenchymal stem cells from Bichat's fat pad: in vitro comparison with adipose-derived stem cells from subcutaneous tissue. Biores Open Access 2013, 2:107-117

22. Shiraishi T, Sumita Y, Wakamastu Y, Nagai $K$, Asahina I: Formation of engineered bone with adipose stromal cells from buccal fat pad. J Dent Res 2012, 91:592-597.

23. Thorwarth M, Schultze-Mosgau S, Kessler P, Wiltfang J, Schlegel KA: Bone regeneration in osseous defects using a resorbable nanoparticular hydroxyapatite. J Oral Maxillofac Surg 2005, 63:1626-1633.

24. Aerssens J, Boonen S, Lowet G, Dequeker J: Interspecies differences in bone composition, density, and quality: potential implications for in vivo bone research. Endocrinology 1998, 139:663-670.

25. Wang S, Liu Y, Fang D, Shi S: The miniature pig: a useful large animal model for dental and orofacial research. Oral Dis 2007, 13:530-537.

26. Terheyden H, Jepsen S, Rueger DR: Mandibular reconstruction in miniature pigs with prefabricated vascularized bone grafts using recombinant human osteogenic protein-1: a preliminary study. Int J Oral Maxillofac Surg 1999, 28:461-463.

27. Nkenke E, Lehner B, Weinzierl $K$, Thams U, Neugebauer J, Steveling H, Radespiel-Troger M, Neukam FW: Bone contact, growth, and density 
around immediately loaded implants in the mandible of mini pigs. Clin Oral Implants Res 2003, 14:312-321.

28. Gahlert M, Gudehus T, Eichhorn S, Steinhauser E, Kniha H, Erhardt W Biomechanical and histomorphometric comparison between zirconia implants with varying surface textures and a titanium implant in the maxilla of miniature pigs. Clin Oral Implants Res 2007, 18:662-668.

29. Wiltfang J, Kloss FR, Kessler P, Nkenke E, Schultze-Mosgau S, Zimmermann R, Schlegel KA: Effects of platelet-rich plasma on bone healing in combination with autogenous bone and bone substitutes in critical-size defects: an animal experiment. Clin Oral Implants Res 2004, 15:187-193.

30. Henkel KO, Gerber T, Dorfling P, Gundlach KK, Bienengraber V: Repair of bone defects by applying biomatrices with and without autologous osteoblasts. J Craniomaxillofac Surg 2005, 33:45-49.

31. Wilson SM, Goldwasser MS, Clark SG, Monaco E, Bionaz M, Hurley WL, Rodriguez-Zas S, Feng L, Dymon Z, Wheeler MB: Adipose-derived mesenchymal stem cells enhance healing of mandibular defects in the ramus of swine. J Oral Maxillofac Surg 2012, 70:e193-e203.

32. Lopa S, De Girolamo L, Arrigoni E, Stanco D, Rimondini L, Baruffaldi Preis FW, Lanfranchi L, Ghigo M, Chiesa R, Brini AT: Enhanced biological performance of human adipose-derived stem cells cultured on titanium-based biomaterials and silicon carbide sheets for orthopaedic applications. J Biol Regul Homeost Agents 2011, 25:S35-S42.

33. Aspenberg $P$, Anttila A, Konttinen $Y T$, Lappalainen $R$, Goodman SB, Nordsletten L, Santavirta S: Benign response to particles of diamond and sic: bone chamber studies of new. Biomaterials 1996, 17:807-812

34. Arrigoni E, Lopa S, de Girolamo L, Stanco D, Brini AT: Isolation, characterization and osteogenic differentiation of adipose-derived stem cells: from small to large animal models. Cell Tissue Res 2009, 338:401-411.

35. Dominici M, Le Blanc K, Mueller I, Slaper-Cortenbach I, Marini F, Krause D, Deans R, Keating A, Prockop D, Horwitz E: Minimal criteria for defining multipotent mesenchymal stromal cells: the International Society for Cellular Therapy position statement. Cytotherapy 2006, 8:315-317.

36. Bourin P, Bunnell BA, Casteilla L, Dominici M, Katz AJ, March KL, Redl H, Rubin JP, Yoshimura K, Gimble JM: Stromal cells from the adipose tissue-derived stromal vascular fraction and culture expanded adipose tissue-derived stromal/stem cells: a joint statement of the International Federation for Adipose Therapeutics and Science (IFATS) and the International Society for Cellular Therapy (ISCT). Cytotherapy 2013, 15:641-648.

37. Tullberg-Reinert $H$, Jundt $G$ : In situ measurement of collagen synthesis by human bone cells with a sirius red-based colorimetric microassay: effects of transforming growth factor beta2 and ascorbic acid 2-phosphate. Histochem Cell Biol 1999, 112:271-276.

38. Halvorsen YD, Franklin D, Bond AL, Hitt DC, Auchter C, Boskey AL, Paschalis EP, Wilkison WO, Gimble JM: Extracellular matrix mineralization and osteoblast gene expression by human adipose tissue-derived stromal cells. Tissue Eng 2001, 7:729-741.

39. Farndale RW, Buttle DJ, Barrett AJ: Improved quantitation and discrimination of sulphated glycosaminoglycans by use of dimethylmethylene blue. Biochim Biophys Acta 1986, 883:173-177.

40. Wolf F, Candrian C, Wendt D, Farhadi J, Heberer M, Martin I, Barbero A Cartilage tissue engineering using pre-aggregated human articular chondrocytes. Eur Cell Mater 2008, 16:92-99.

41. Schwarz C, Leicht U, Rothe C, Drosse I, LuibI V, Rocken M, Schieker M: Effects of different media on proliferation and differentiation capacity of canine, equine and porcine adipose derived stem cells. Res Vet Sci 2012, 93:457-462

42. Im Gl, Ahn JH, Kim SY, Choi BS, Lee SW: A hyaluronate-atelocollagen/ beta-tricalcium phosphate-hydroxyapatite biphasic scaffold for the repair of osteochondral defects: a porcine study. Tissue Eng Part A 2010, 16:1189-1200.

43. Zheng Y, Liu Y, Zhang CM, Zhang HY, Li WH, Shi S, Le AD, Wang SL: Stem cells from deciduous tooth repair mandibular defect in swine. J Dent Res 2009, 88:249-254.

44. de Girolamo L, Lopa S, Arrigoni E, Sartori MF, Baruffaldi Preis FW, Brini AT: Human adipose-derived stem cells isolated from young and elderly women: their differentiation potential and scaffold interaction during in vitro osteoblastic differentiation. Cytotherapy 2009, 11:793-803.

45. Song SH, Kumar BM, Kang EJ, Lee YM, Kim TH, Ock SA, Lee SL, Jeon BG, Rho GJ: Characterization of porcine multipotent stem/stromal cells derived from skin, adipose, and ovarian tissues and their differentiation in vitro into putative oocyte-like cells. Stem Cells Dev 2011, 20:1359-1370.

46. Noort WA, Oerlemans MI, Rozemuller H, Feyen D, Jaksani S, Stecher D, Naaijkens B, Martens AC, Buhring HJ, Doevendans PA, Sluijter JP: Human versus porcine mesenchymal stromal cells: phenotype, differentiation potential, immunomodulation and cardiac improvement after transplantation. J Cell Mol Med 2012, 16:1827-1839.

47. Pei D: Regulation of pluripotency and reprogramming by transcription factors. J Biol Chem 2009, 284:3365-3369.

48. De Girolamo L, Stanco D, Salvatori L, Coroniti G, Arrigoni E, Silecchia G, Russo MA, Niada S, Petrangeli E, Brini AT: Stemness and osteogenic and adipogenic potential are differently impaired in subcutaneous and visceral adipose derived stem cells (ASCs) isolated from obese donors. Int I Immunopathol Pharmacol 2013, 26:11-21.

49. Qu CQ, Zhang GH, Zhang LJ, Yang GS: Osteogenic and adipogenic potential of porcine adipose mesenchymal stem cells. In Vitro Cell Dev Biol Anim 2007, 43:95-100.

50. Tang L, Yin Y, Zhou H, Song G, Fan A, Tang B, Shi W, Li Z: Proliferative capacity and pluripotent characteristics of porcine adult stem cells derived from adipose tissue and bone marrow. Cell Reprogram 2012, 14:342-352

51. Im W, Chung JY, Kim SH, Kim M: Efficacy of autologous serum in human adipose-derived stem cells; cell markers, growth factors and differentiation. Cell Mol Biol 2011, 57:OL1470-OL1475.

52. Choi J, Chung JH, Kwon GY, Kim KW, Kim S, Chang H: Effectiveness of autologous serum as an alternative to fetal bovine serum in adipose-derived stem cell engineering. Cell Tissue Bank 2012, 14:413-422.

53. Kurita M, Aiba-Kojima E, Shigeura T, Matsumoto D, Suga $H$, Inoue K, Eto $H$, Kato $\mathrm{H}$, Aoi N, Yoshimura K: Differential effects of three preparations of human serum on expansion of various types of human cells. Plast Reconstr Surg 2008, 122:438-448.

54. Kuznetsov SA, Mankani MH, Robey PG: Effect of serum on human bone marrow stromal cells: ex vivo expansion and in vivo bone formation. Transplantation 2000, 70:1780-1787.

55. Yamamoto N, Isobe M, Negishi A, Yoshimasu H, Shimokawa H, Ohya K Amagasa T, Kasugai S: Effects of autologous serum on osteoblastic differentiation in human bone marrow cells. J Med Dent Sci 2003, 50:63-69.

56. Mizuno N, Shiba H, Ozeki Y, Mouri Y, Niitani M, Inui T, Hayashi H, Suzuki K, Tanaka S, Kawaguchi $H$, Kurihara H: Human autologous serum obtained using a completely closed bag system as a substitute for foetal calf serum in human mesenchymal stem cell cultures. Cell Biol Int England 2006, 30:521-524.

57. Shahdadfar A, Fronsdal K, Haug T, Reinholt FP, Brinchmann JE: In vitro expansion of human mesenchymal stem cells: choice of serum is a determinant of cell proliferation, differentiation, gene expression, and transcriptome stability. Stem Cells 2005, 23:1357-1366.

58. Farre-Guasch E, Marti-Page C, Hernadez-Alfaro F, Klein-Nulend J, Casals N: Buccal fat pad, an oral access source of human adipose stem cells with potential for osteochondral tissue engineering: an in vitro study. Tissue Eng Part C Methods 2010, 16:1083-1094.

\section{doi:10.1186/scrt359}

Cite this article as: Niada et al:: Porcine adipose-derived stem cells from buccal fat pad and subcutaneous adipose tissue for future preclinical studies in oral surgery. Stem Cell Research \& Therapy 2013 4:148. 